\title{
Experiment and application of soft $x-$ ray grazing incidence optical scattering phenomena
}

Shuyan Chen, Cheng Li, Yang Zhang, Liping Su, Tao Geng, et al.

Shuyan Chen, Cheng Li, Yang Zhang, Liping Su, Tao Geng, Kun Li, "Experiment and application of soft x-ray grazing incidence optical scattering phenomena," Proc. SPIE 10452, 14th Conference on Education and Training in Optics and Photonics: ETOP 2017, 104526G (16 August 2017); doi: $10.1117 / 12.2269945$

SDIE Event: 14th Conference on Education and Training in Optics and Photonics, ETOP 2017, 2017, Hangzhou, China 


\title{
Experiment and Application of Soft X-ray Grazing Incidence Optical Scattering Phenomena
}

\author{
Shuyan Chen, Li Cheng, Yang Zhang", Liping Su, Tao Geng, Kun Li \\ Key Laboratory of integrated fiber optics, Ministry of education. National Demonstration Center for \\ Physical Experiment Education, Harbin Engineering University, China.
}

\begin{abstract}
For short wavelength imaging systems, surface scattering effects is one of important factors degrading imaging performance. Study of non-intuitive surface scatter effects resulting from practical optical fabrication tolerances is a necessary work for optical performance evaluation of high resolution short wavelength imaging systems. In this paper, Soft X-ray optical scattering distribution is measured by a soft X-ray reflectometer installed by my lab, for different sample mirrors、 wavelength and grazing angle. Then aim at space solar telescope, combining these scattered light distributions, and surface scattering numerical model of grazing incidence imaging system, PSF and encircled energy of optical system of space solar telescope are computed. We can conclude that surface scattering severely degrade imaging performance of grazing incidence systems through analysis and computation.
\end{abstract}

Keywords: Soft X-ray, Grazing Incidence, Optical Scattering Phenomena, Experiment

*Third Author E-mail: zhangyang@hrbeu.edu.cn

\section{INTRODUCTION}

With the development of the space times, the space technology is becoming more and more dependent on the space technology. The solar activity directly affects and restricts the change of the near earth space environment ${ }^{1,2}$. The characteristics of soft X-ray band are more obvious when the solar bursts occur. Therefore, it is of great significance to carry out the soft X-ray observation to predict the solar activity and space weather. In the observation of the solar spectrum, the soft X-ray band imaging observation has a unique advantage in the development of space weather forecast. Study on the soft X-ray imaging system, not only can help the change of human monitoring of the space environment, ensure the production safety of life, but also can help people further understand the mysteries of the universe, the universe provides important data. At present, the research of grazing incidence space soft X-ray imaging telescope in our country is still in the initial stage and has not been put into operation ${ }^{3,4}$. Based on the importance of space environment monitoring, it is urgent to research and develop the space soft X-ray telescope. The grazing incidence optical system has the advantages of wide range of working wavelength, and so on. In the soft X-ray band, the commonly used grazing incidence telescope is Wolter-I telescope. For this optical system, effective light area and surface scattering are two important factors that affect the imaging quality ${ }^{5-8}$. This paper focuses on the space of soft X-ray grazing incidence optical system, from the aspects of smooth surface scattering, soft X-ray reflectivity we developed the application respectively for mirrors with different samples, experiments were carried out to study the surface scattering of optical radiation soft X-ray band in different bands of grazing and grazing angle under the condition of. After data processing, respectively, analyzed the influence of grazing angle, wavelength and roughness factors of grazing incidence soft X-ray surface scattering from the experimental point of view; On the other hand, start from the application, space solar telescope optical system soft X-ray image quality evaluation, discuss the influence of optical surface scattering on the quality of soft X-ray grazing incidence imaging optical system?

\section{SOFT X-RAY GRAZING INCIDENCE OPTICAL SCATTERING EXPERIMENTATION}

On the surface scattering experiment, influence of surface scattering upon reflector is incarnated on the effective area. Based on the existing conditions of $\mathrm{LAB}$, data of optical scattered distribution are obtained by soft X-ray reflectometer. The soft X-ray reflectometer consists of a laser-produced-plasma (LPP) light source with gas-jet target, a monochromator, a high vacuum sample chamber, a vacuum pumping system and an electronics unit shown in Fig.1.

Laser plasma light source produced by the soft X-ray continuous spectrum after grazing incidence of constant Angle monochromator, select the required working wavelength of monochromatic light, and then enter indoor sample;

14th Conference on Education and Training in Optics and Photonics: ETOP 2017, edited by Xu Liu,

Xi-Cheng Zhang, Proc. of SPIE Vol. 10452, 104526G · @ 2017 ICO, IEEE, OSA, SPIE

CCC code: $0277-786 \mathrm{X} / 17 / \$ 18 \cdot$ doi: $10.1117 / 12.2269945$

Proc. of SPIE Vol. 10452 104526G-1 
Monochromatic soft X-ray after mirror reflection under test by channel electron multiplier detection, probe detection results by general computer output by data acquisition system, realize the surface scattering measurement. The following is the specific content of grazing incidence optical surface scattering experiment respectively discussed, first of all samples selected from the beginning.

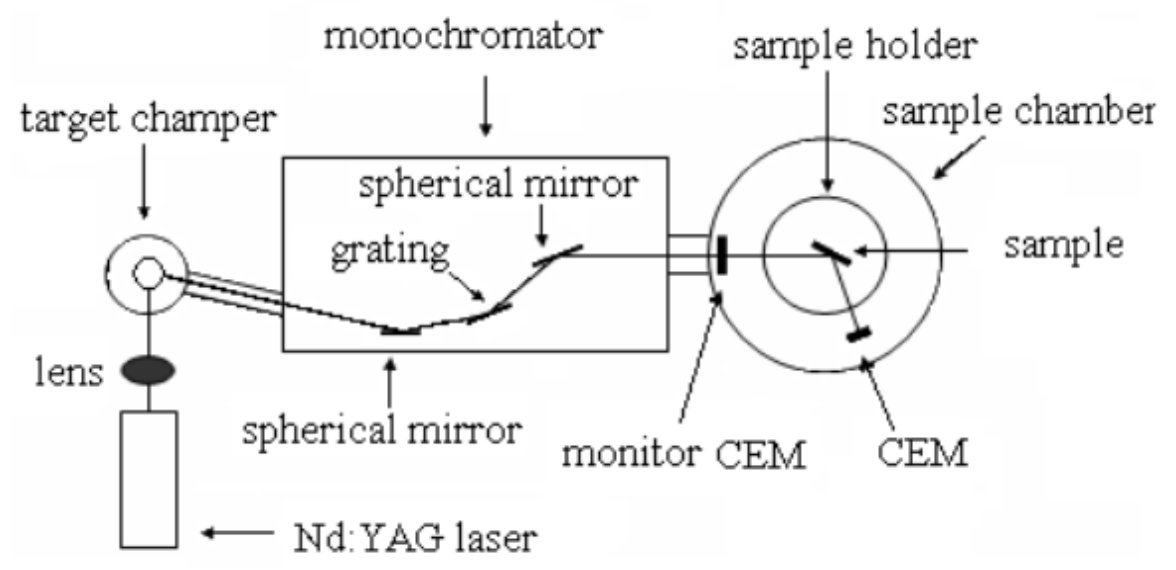

Fig.1: Schematic diagram of the soft X-ray reflectometer

\subsection{Sample}

In this experiment, according to the optical materials and surface morphology, choose two different samples of mirror. Two pieces of samples are flat mirror, one is a piece of microcrystalline glass material, and another piece of sample is silicon. Microcrystalline glass with low thermal expansion coefficient, good thermal stability, dimension stability and high mechanical strength and become a large optical instrument mirror of high quality materials; Silicon is a kind of commonly used optical lens materials. Before the surface scattering experiment, we first adopt WYKO surface contourgraph respectively on the surface roughness of the two pieces of test samples determined, the results of the determination of as shown in figure.2, which (a) of silicon mirror under test samples, (b) as microcrystalline glass mirror under test samples. As can be seen from the two diagrams, silicon overall surface roughness RMS values of the sample under test is $1.3 \mathrm{~nm}$, and microcrystalline glass overall roughness RMS values of the sample under test is $1.5 \mathrm{~nm}$.

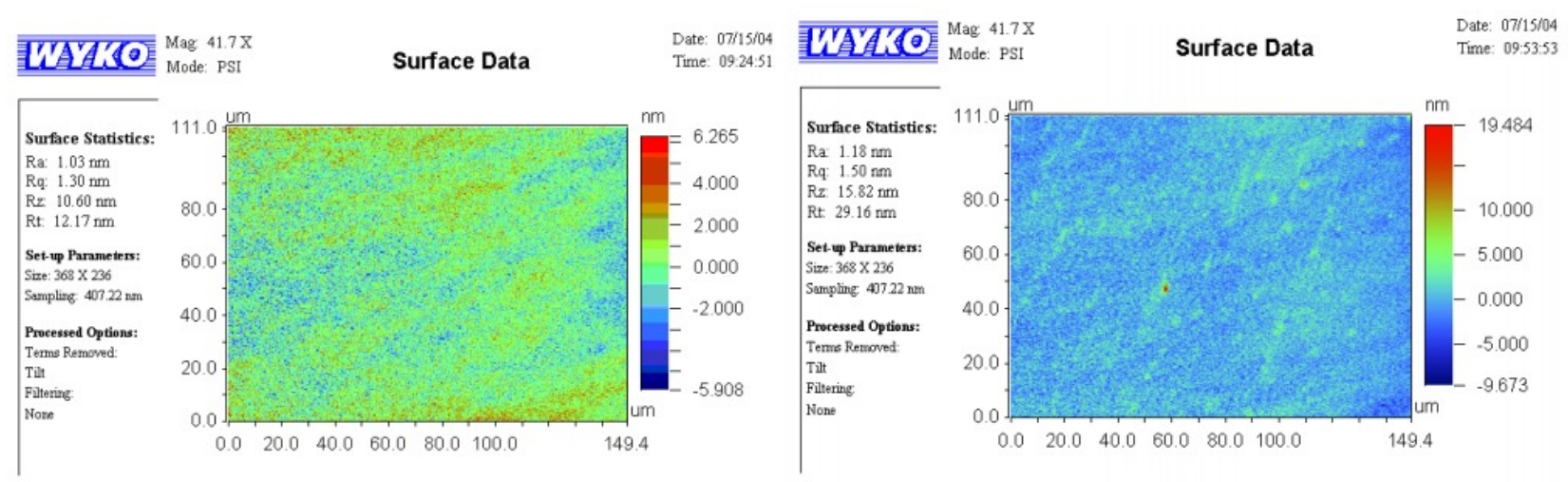

Fig.2(a): Surface data of Si sample by WYKO

Fig.2(b): Surface data of glass-ceramics sample by WYKO

\subsection{Different factors effects on surface scattering}

In experiment, the first measured surface scattering Angle distribution of different wavelengths, namely for the same sample under test, on the basis of the grazing angle, the surface of different wavelength of incident light scattering experiment; Change the grazing Angle, then continue to measure the surface scattering distribution in the different 
wavelengths; When several after the grazing Angle measured, turn off the system, change different sample under test, the vacuum, high pressure until at least $1000 \mathrm{v}$, repeat the previous experimental process of the sample under test. By using these scattered light distributions of super-smooth surfaces measured by the soft X-ray reflectometer, for different sample mirrors、 wavelength and grazing angle. By dealing with experimental data, different factors effects on surface scattering, such as glancing angle、wavelength and roughness is computed and analyzed.

\subsubsection{Wavelength}

For soft X-Ray grazing incidence optical surface scatter do not satisfied approximation of smooth surface, so there is no directly proportion between wavelength and surface scattering, and these relation will be analyzed by experimentation. Based on compute process of surface roughness, we get effective scattering distribution and incidence distribution of glass-ceramics sample while wavelength is $8 \mathrm{~nm} 、 9 \mathrm{~nm} 、 10 \mathrm{~nm} 、 10 \mathrm{~nm}$ and grazing incidence angle is $1.5^{\circ}$, shown in Fig. 3(a), and effective scattering distribution and incidence distribution of Si sample while wavelength is $13 \mathrm{~nm} 、 14 \mathrm{~nm}$ 、 $15 \mathrm{~nm} 、 16 \mathrm{~nm}$ and grazing incidence angle is $1.5^{\circ}$, shown in Fig. 3(b). From Fig.3, same surface roughness, same grazing angle, along with decrease of wavelength, optical surface scatter is more serious, and reduce performance of optics, and then we can see that for short wavelength optical system, optical surface scattering research is very important.

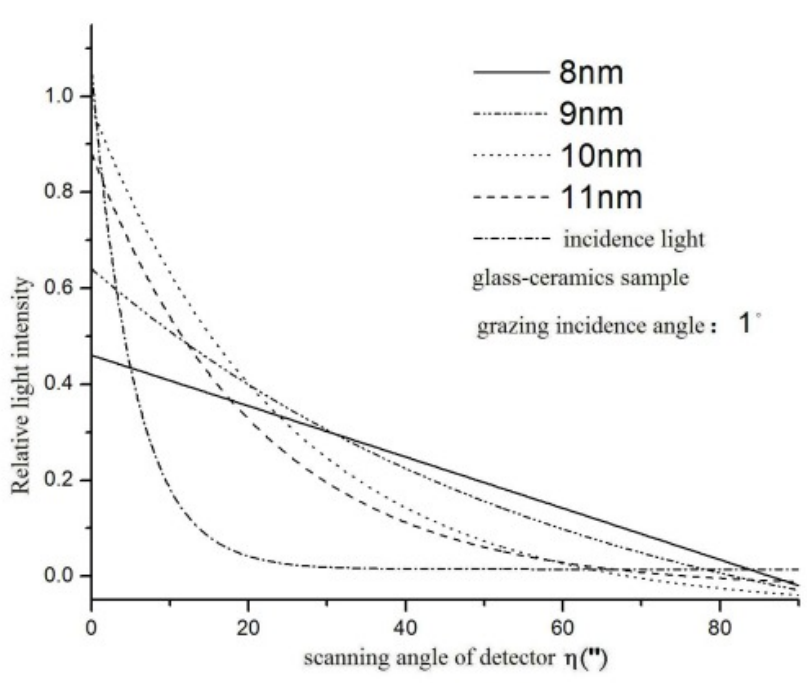

Fig.3(a): $\phi=1^{\circ}$ effective scattering distribution of glass-ceramics sample with different wavelength

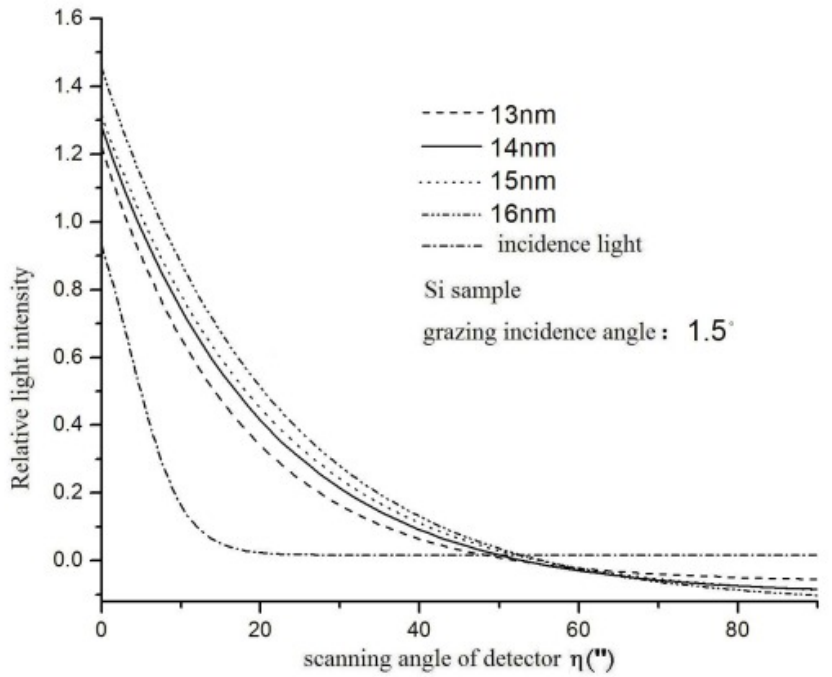

Fig.3(b): $\phi=1.5^{\circ}$ effective scattering distribution of Si sample with different

\subsubsection{Surface roughness}

Effective scattering distribution and incidence distribution of Si sample and glass-ceramics sample is shown in Fig. 4(a) while wavelength is $8 \mathrm{~nm}$ and grazing incidence angle is $1^{\circ}$, where abscissa is scanning angle of detector $\eta$ (difference of

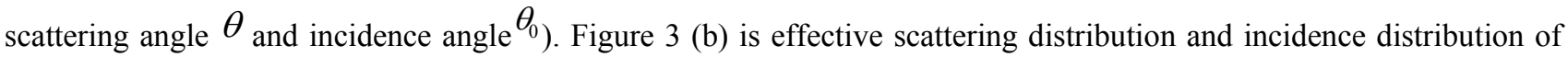
two pieces of samples while wavelength is $11 \mathrm{~nm}$, grazing Angle is $1.5^{\circ}$.

From Fig.4, we can see the silicon sample scattering degree is not as serious as the scattering degree of microcrystalline glass sample, and from the previous figure 2, we know surface roughness of silicon samples is smaller than the microcrystalline glass samples. Therefore, we can come to a conclusion that is in the soft X-ray band, with the increase of surface roughness, surface of grazing incidence optical scattering is more and more serious. In that case, we fully proved that increasing the surface roughness through the experiment can aggravate the degree of grazing incidence optical surface scattering, the serious influence the image quality of grazing incidence optical imaging system. 


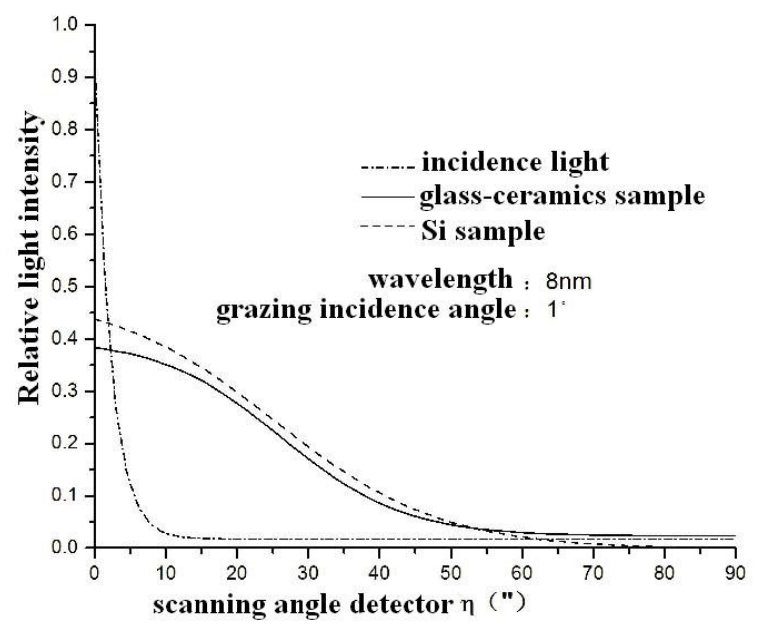

Fig.4(a): $\lambda=8 \mathrm{~nm} 、 \quad \phi=1^{\circ}$ effective scattering distribution and incidence distribution with different roughness

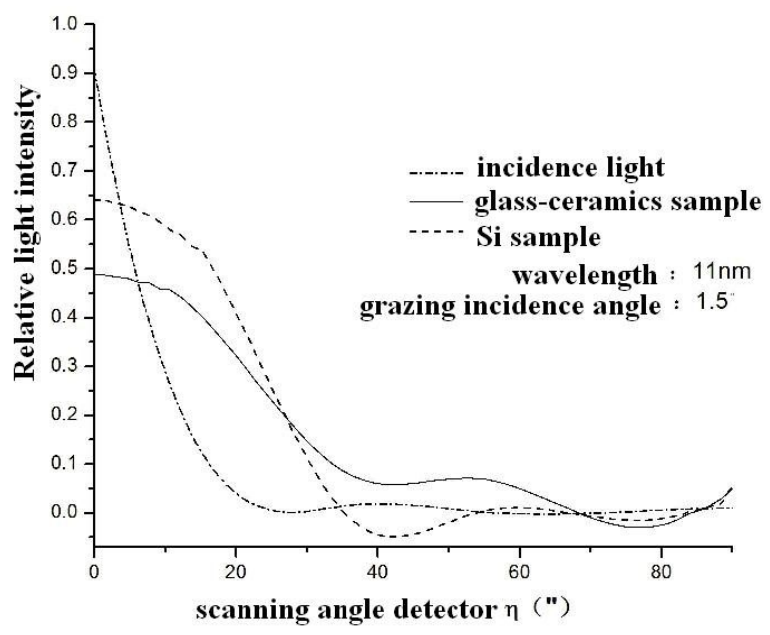

Fig.4(b): $\lambda=11 \mathrm{~nm} 、 \quad \phi=1.5^{\circ}$ effective scattering distribution and incidence distribution with different roughness

\subsubsection{Grazing angle}

Grazing angle is complementary angle of incidence angle, so grazing angle affection is same with incidence angle affection discussed. Grazing angle is in the phase function of pupil function, has influence on the scattering distribution. Effective scattering distribution and incidence distribution of glass-ceramics sample while wavelength is $9 \mathrm{~nm}$ and grazing incidence angle is $1^{\circ} 、 1.5^{\circ} 、 2^{\circ}$, is shown in Fig.5(a), and effective scattering distribution and incidence distribution of Si sample while wavelength is $11 \mathrm{~nm}$ and grazing incidence angle is $1^{\circ} 、 1.5^{\circ} 、 2^{\circ}$, is shown in Fig.5(b). From Fig.5, same surface roughness, same wavelength, along with increase of grazing angle, optical surface scatter is more serious, and reduce performance of optics, and then we can improved optical performance through decrease grazing angle.

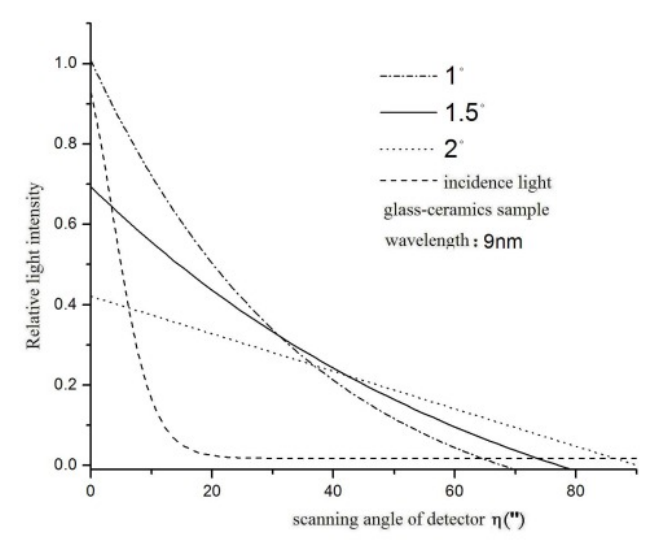

Fig.5(a): $\lambda=9 \mathrm{~nm}$ effective scattering distribution of glass-ceramics sample with different grazing angle

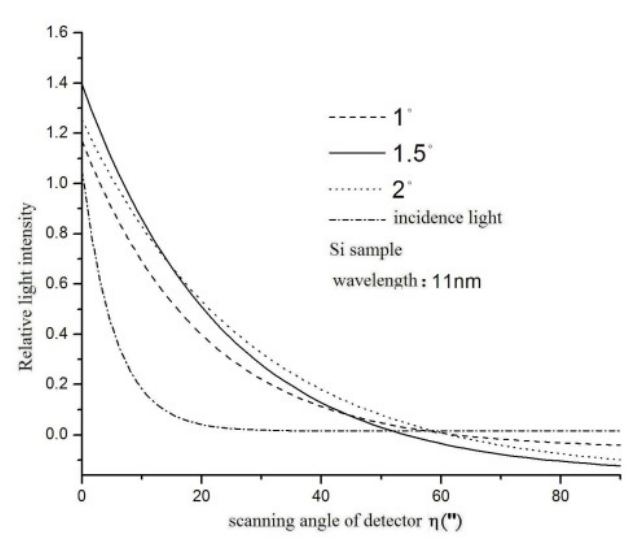

Fig.5(b): $\lambda=11 \mathrm{~nm}$ effective scattering distribution of $\mathrm{Si}$ sample with different grazing angle

Above, from the Angle of the experiment, we have analyzed respectively the influence of grazing Angle, wavelength and surface roughness on soft X-ray grazing incidence scattering, we will discuss below application of Soft X-ray Grazing Incidence Optical Scattering Phenomena. 


\section{APPLICATION: EFFECTS OF SURFACE SCATTERING TO IMAGING PERFORMANCE OF SPACE SOLAR TELESCOPE}

Aperture diffraction、 geometric aberrations、surface scatter and other errors (setting errors、 environmental errors) are several influencing factors on imaging performance of optical system. Especially for short wavelength imaging systems, Surface scattering effects is one of important factors degrading imaging performance. Aimed at Wolter- I grazing incidence optical system of space solar telescope, by combining those scattered light distributions of super-smooth surfaces measured, and surface scattering mathematical model of space solar telescope grazing incidence imaging system, effects of surface scattering to imaging performance of space solar telescope will be evaluated then, and applying of surface scattering in imaging evaluation of soft X-ray grazing incidence optical system will be discussed.

Distribution of effective scattering is shown in Fig.5 while wavelength is $16 \mathrm{~nm}$ and grazing incidence angle is $1.5^{\circ}$, where abscissa is scanning angle of detector. Scattering light distribution shown in above Fig.6(a) is convolution of incidence light distribution and sample scatter PSF

$$
I=S * I \text { i nci dent } \mathrm{l} \text { ight }
$$

Based on effective scattering distribution and incidence distribution of glass-ceramics sample while wavelength $\lambda=$ $16 \mathrm{~nm} 、 \phi=1.5^{\circ}$ ( shown in Fig. 6(a)), through deconvolution, we can obtain angle spread function and encircled energy of optical system of space solar telescope are obtained, shown in Fig.6(b), 6 (c), 6 (d). from Fig. 6 (c), Radius of scatter facula covered $63 \%$ energy is 19 " through computing. If now image core is calculated, except surface scatter, effects of aperture diffraction geometric aberrations and other errors are calculated also, we can get encircled energy of space solar telescope, shown in Fig.6(d), and radius of light facula covered 63\% energy is $22 "$.

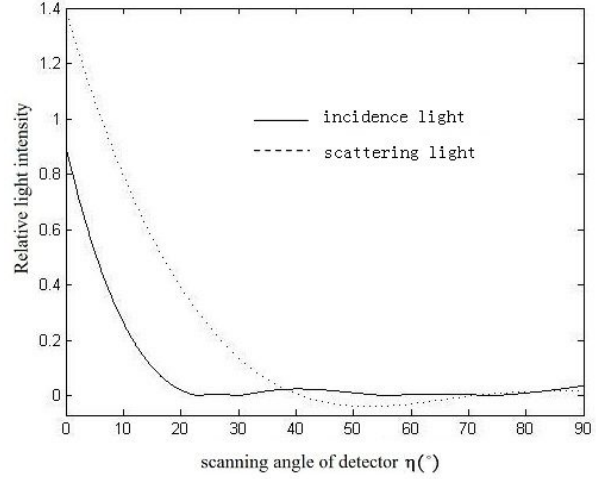

Fig.6(a): $\lambda=16 \mathrm{~nm}, \quad \phi=1.5^{\circ} \quad$ effective scattering distribution of glass-ceramics sample distribution

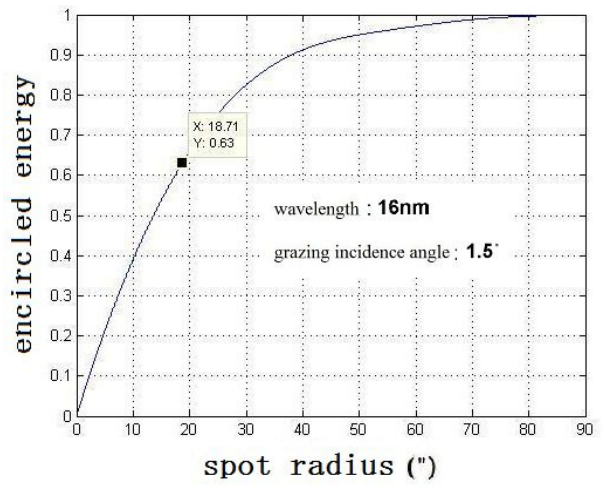

Fig.6(c): dimension of image core is zero,encircled energy Of space solar telescope

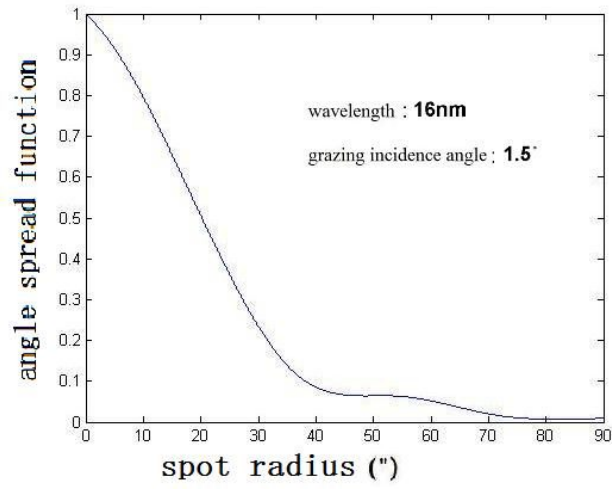

Fig.6(b): $\lambda=16 \mathrm{~nm}, \phi=1.5^{\circ}$ angle spread function of space solar telescope

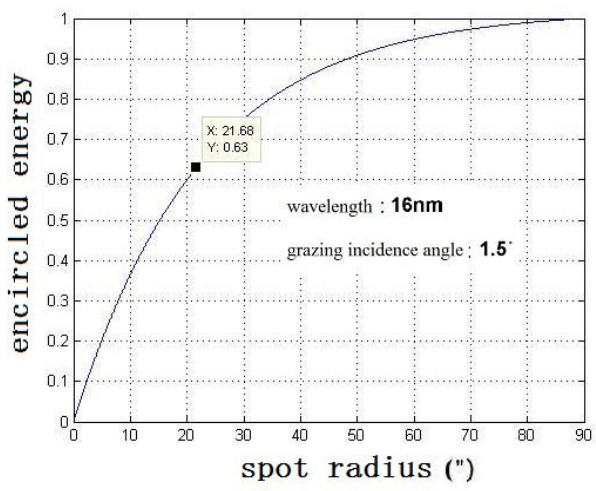

Fig.6(d): $\lambda=16 \mathrm{~nm}, \phi=1.5^{\circ}$ encircled energy Of space solar telescope 


\section{CONCLUSION}

Based on the analysis of experimental results the parameters such as surface roughness, wavelength and grazing Angle influence on grazing incidence surface scattering, research has shown that with the increase of surface roughness, grazing Angle increase and the decrease of the wavelength, grazing incidence optical scattering phenomenon is more and more serious. And aimed at Wolter- I grazing incidence optical system of space solar telescope, by combining those scattered light distributions of super-smooth surfaces measured, and surface scattering mathematical model of space solar telescope grazing incidence imaging system, we evaluated effects of surface scattering to imaging performance of space solar telescope. From point spread function and encircled energy of solar telescope optical system, we got a conclusion that Surface scattering is one of the main factors influencing the grazing incidence telescope image quality.

\section{REFERENCES}

[1] Bake. D. N, What is space weather [J], Adv. Space Res.22, 7-16(1998).

[2] Atmos, Solar-Terr, Phys.62, 1669-1681(2000).

[3] J. E. Harvey and Cynthia L.Vernold, Transfer function characterization of scattering surfaces: Revisited, Proc. SPIE .3141, 113-127(1976).

[4] J. E. Harvey, Light-scattering characteristics of optical surfaces, Ph.D. Dissertation, U. Arizona(1976).

[5] Chen.B, Ni.Q.L, Wang.J.L, soft X-ray and extreme ultraviolet optics in CIOM, Optics and Precision Engineering. 15, 1863-1868 (Dec 2007).

[6] Meng.Y.L, Wang.Y.G, Chen.S.Y, Chen.B, Assessment of surface roughness by use of soft x-ray scattering,SPIE.v7451, (2009).

[7] Chen.S.Y, Xu.X.J, Qi.L.H, Measurement and analysis of surface profiles by inverse scattering method, SPIE.v 7656, n PART 1(2010).

[8] Wolter.H, Mirror systems with glancing incidence on imaging producing optics for X-ray[J], Ann Phys. 94(10), 866-875(1952).

[9] Yang.Z, Dai.Y.F, W.G.L, Measure of Optical Errors Based on Harvey-Shack Surface Scattering Theory, ACTA OPTICA SINICA . 27(11), 1992-1996 (2007) 